\title{
Experience and lessons from the use of lepromin and Mycobacterium leprae-specific serology
}

\author{
U. SENGUPTA \\ Central JALMA Institute for Leprosy, Tajganj, Agra, India
}

\begin{abstract}
Summary Skin testing with lepromin, which produces a delayed-type hypersensitivity reaction, has been used in the classification of leprosy, and a good correlation has been found between immunological status and the reaction to lepromin. In addition, the prognostic value of the lepromin test has been demonstrated. More recently, skin testing with two soluble antigens of Mycobacterium leprae showed no difference of the mean size of the reaction between household contacts and noncontacts, indicating that these antigens are not useful for the diagnosis of leprosy. This and other evidence points to the need for a better skin test antigen capable of detecting infection of individuals by $M$. leprae. Whereas serological assays for antibodies against both PGL- 1 and the $35 \mathrm{kDa}$ antigen of $M$. leprae have been found to yield positive results in $90-100 \%$ of patients with lepromatous (BL/LL) leprosy, these assays fail to identify $40-60 \%$ of patients with tuberculoid (BT/TT) leprosy, because of the presence of only an insignificant level of antibody against components of $M$. leprae in these patients' serum, although, in many BT patients, antibody signal could be detected in the local lesions. These data indicate that there remains a need for a specific diagnostic test for leprosy.
\end{abstract}

\section{Introduction}

Skin tests and serological tests specific for Mycobacterium leprae, which could serve as tools fordetecting infection by the organism in a population at risk of developing leprosy, represent basic needs for epidemiological study. This critical review assesses whether the tests that have been developed in the past satisfy these needs.

\section{Lepromin}

The lepromin test, developed in 1916 by Mitsuda, ${ }^{1}$ was later redefined and ultimately standardized by Hanks and coworkers, ${ }^{2}$ who recommended a concentration of M. leprae of $1.6 \times 10^{8}$ organisms per ml. Mitsuda lepromin has been used to elicit a nodular skin reaction (the 'late reaction') 3-4 weeks after intradermal inoculation of $0 \cdot 1 \mathrm{ml}$ of the material. Later, Mitsuda lepromin was also recommended for use in a concentration of $4 \times 10^{7} \mathrm{M}$. leprae per $\mathrm{ml}^{3}$

In 1942, Dharmendra ${ }^{4}$ prepared a defatted preparation of $M$. leprae obtained by extraction of a leproma rich in $M$. leprae with chloroform and ether. This preparation was initially standardized by suspending $1 \mathrm{mg}$ of the dried extract in $10 \mathrm{mg} 0.5 \%$ carbol saline. Intradermal 
inoculation of $0.1 \mathrm{ml}$ of this material elicited only the 'early reaction' (24-48 h). Because various standardized batches showed variability in evoking a skin reaction, the antigen was further standardized to contain $10^{7}$ bacilli per ml of the preparation. ${ }^{5}$ This antigen was capable of inducing not only the early reaction but also the late reaction.

After $M$. leprae became available from the armadillo, soluble antigens were prepared by extracting purified $M$. leprae obtained either by sonication of irradiation-killed organisms, followed by centrifugation to remove cell walls and standardization at a concentration of $1 \cdot 0 \mu \mathrm{g}$ protein per $0 \cdot 1 \mathrm{ml}$ (the Rees antige ${ }^{5}$ ), or by disruption of irradiation-killed $M$. leprae in a French press, followed by filtration to remove cell walls, autoclaving and adjustment to a concentration of $0.5 \mu \mathrm{g}$ protein per $\mathrm{ml}$ (the Convit antigen ${ }^{6}$ ). Both Rees and Convit antigens elicit an early reaction.

All of these skin test antigens have been used extensively in both endemic and nonendemic populations. Generally, an area of induration at least $10 \mathrm{~mm}$ in diameter has been taken as an early reaction, and a nodular reaction greater than $4 \mathrm{~mm}$ in diameter has been taken as positive for the late reaction. However, Gupte and coworkers ${ }^{7}$ considered only a skin reaction of at least $12 \mathrm{~mm}$ as a positive early reaction. The cut-off point used by different workers has varied extensively, ranging from $2 \mathrm{~mm}^{7}$ to more than $10 \mathrm{~mm}$. ${ }^{9}$ These differences in cut-off point may have been arbitrary, or they may have resulted from differences in preparation of the antigens, or differences in the populations with respect to sensitization by environmental mycobacteria. ${ }^{10}$

The prognostic value of the late lepromin reaction among healthy individuals in an endemic population was demonstrated by Dharmendra and Chatterjee, ${ }^{11}$ who showed that the risk of leprosy was greater among lepromin-negative individuals. Among these leprominnegative individuals, lepromatous leprosy was more commonly observed in persistently negative individuals.

The lepromin test has been used to determine the immune status of the individual, and for monitoring changes of the patients' immune status during treatment. It has been universally observed that, along the leprosy spectrum, the lepromin test remains negative in lepromatous patients, whereas a positive reaction is found in the majority of tuberculoid patients. ${ }^{6,12}$

However, the use of lepromin to determine the proportion of a large population sensitized to $M$. leprae was investigated only recently, when lepromin was employed in vaccine trials. Pönnighaus and Fine ${ }^{10}$ and Gupte et al., ${ }^{7}$ noted that the size of skin reaction was biomodally distributed in the population, similar to the distribution of the skin reaction to tuberculin. There was no difference of the size of reaction between the patients, their contacts and the healthy population in the same field area, indicating that, as a skin test antigen, lepromin fails to discriminate between healthy individuals and patients. Further, variability was noted in the size of skin reactions to different batches of the same type of lepromin. ${ }^{13}$

These data strongly indicate that presently available lepromin preparations are not capable of identifying those healthy persons who are infected with $M$. leprae in a population in which leprosy is endemic. A better antigen is required for identifying the M. lepraeinfected in a healthy population, particularly in a situation in which other cross-reactive mycobacterial antigens might be important in cross-sensitization of the healthy population.

\section{Specific serology}

Several serological assays thought to detect antibodies specific to $M$. leprae have been 
developed. However, only two, one detecting anti-PGL-1 antibodies, and one detecting antibodies to the $35 \mathrm{kDa}$ epitope, have been felt to be sufficiently specific and reproducible to be used extensively. These assays have been found capable of diagnosing $90-100 \%$ of active cases of advanced leprosy, but have failed to identify $40-60 \%$ of early active cases of leprosy. ${ }^{14}$ In fact, it was noted that the sera of the majority of TT/BT patients did not even contain significant levels of antibody against whole $M$. leprae (unpublished observation).

We noted that suspensions of skin scrapings from BT/TT lesions were positive for M. leprae $35 \mathrm{kDa}$ antibody. ${ }^{15}$ To study the local immune response, we developed ${ }^{15,16}$ an organotypic culture of skin, and studied the secretions of cultures of TT/BT skin lesions. We found that the kinetics of the antibody response to $M$. leprae could be easily studied in the lesions; also, many of the TT/BT lesions were found to be positive for antibody. However, skin scrapings obtained from patients with vitiligo were also found to be positive for the $35 \mathrm{kDa}$ antibody, indicating cross-reactivity.

\section{Conclusion}

It is clear that a test to detect the M. leprae-infected in the community does not yet exist. Therefore, development should be undertaken of a specific test, either a skin test or a serological test, for leprosy that can discriminate between infection by $M$. leprae and a reaction to environmental mycobacteria.

\section{References}

${ }^{1}$ Hayashi F. Mitsuda's skin reaction. Int J Lepr, 1933; 1: 31-38.

2 Hanks JH, Abe M, Nakayama T et al. Studies toward the standardization of lepromin. Bull WHO, 1970; 42: 703709.

${ }^{3}$ World Health Organization. Recommended safety requirements for the preparation of lepromin. Bull WHO, 1979; 57: $921-923$

${ }^{4}$ Dharmendra. Studies of the lepromin test (9). A bacillary antigen standardized by weight. Lepr Ind, 1942; 14: $122-129$.

5 Sengupta U, Ramu G, Desikan KV. Assessment of Dharmendra Antigen. II. Standardization of the antigen. Lepr Ind, 1979; 51: 316-322.

${ }^{6}$ World Health Organization. Vaccination trials against leprosy. Meeting of the Epidemiology Subgroup of Scientific Working Group on the Immunology of Leprosy, Geneva, 11-13 February 1985; TDR/IMMLEP/EPD/ 85.3: pp 7-8.

7 Gupte MD, Anantharaman D, Nagaraju B, Kannan, S, Vallishayee RS. Experiences with Mycobacterium leprae soluble antigens in a leprosy endemic population. Lepr Rev, 1990; 61: 132-144.

8 Stanford JL, Lema E. The use of a sonicate preparation of Mycobacterium tuberculosis (new tuberculin) in assessment of BCG vaccination. Tubercle, 1983; 64: 275-282.

9 Zuniga M. Evolution of the leprosy endemic in the Americas, and projections for the year 2000. Working paper for WHO Study Group meeting on 'Epidemiology of Leprosy in Relation to Control', Geneva, 1983.

10 Pönnighaus JM, Fine PEM. (1986). Sensitization studies with potential leprosy vaccine preparations in northern Malawi. Int J Lepr, 1986; 54: 25-37.

11 Dharmendra, Chatterjee KR. Prognostic value of lepromin test in contacts of leprosy cases. Lepr India, 1955; 27: 149-152.

12 Samuel NM, Stanford JL, Rees RJW et al. Human vaccination studies in normals and contacts of leprosy patients. Ind J Lepr, 1984; 56: 36-47.

13 Gupte MD, Anantharaman DS. Use of soluble antigens in leprosy epidemiology. Lepr Rev, 1988; 59: 329335.

${ }^{14}$ Sengupta U. Mycobacterium leprae antigens and their utility in immunodiagnostics of leprosy Trop Med Parasit, 1990; 41: 361-362. 
15 Parkash Om, Beuria MK, Girdhar BK et al. Effort in diagnosing early leprosy using serological techniques. $J$ Biosciences, 1997; 22: 111-116.

16 Sengupta U, Beuria MK, Mohanty KK, Katoch K. Serological diagnosis of leprosy using full thickness skin cultures in vitro: a preliminary study. Tenth International Congress of Immunology. New Delhi, November 1-6, 1998, eds Talwar GP, Nath I, Ganguly NK, Rao KVS. International Proceedings Division, Monduzzi Editore, Liosei-Rastignano-Bologna, Italy, 1998, pp 793-796. 